\title{
NETWORK CENTRIC SATELLITE COMMUNICATION WITH IP NETWORKS BASED ON REGENERATIVE SATELLITE MESH-A (RSM-A) KA BAND TECHNOLOGY
}

\author{
Rajeev Gopal, Steve Arnold \\ Hughes Network Systems, LLC \\ Germantown, MD
}

\section{ABSTRACT}

Network centric unification of diverse communication and information sharing systems is a key objective for national defense. Overall, IP-convergence is the basis for seamlessly integrating the DoD SATCOM networking and information needs with the Global Information Grid (GIG). This paper explores IP networking with the emerging Regenerative Satellite Mesh-A (RSM-A) Ka band technology. The focus is on RSM-A capabilities to address key network centric requirements in providing reach back and range extension capabilities. Hughes RSM-A implementation's key architectural features, with on-board packet processing and bandwidth on demand, facilitate full-mesh, multi Gbps IP packet processing SATCOM. The relevant RSM-A based capabilities and services are described with respect to a framework for evaluating satellite system architectures for meeting network centric requirements. This framework analyzes selected networking functions required by the end users and how they are supported at the various network layers in satellite communication system segments. This analysis is illustrated with conceptual design and architectural attributes, supported by representative data from test results and recent operational experience over a commercial satellite system based on RSM-A.

\section{INTRODUCTION}

Satellite networking is reaching an inflection point, facilitated by net-centric architectural influences, for larger coverage, capacity, and ease of deployment. The role of IP protocol is central to efficient inter-operability across diverse wired and radio transport technologies for both terrestrial and space components. Satellite networks are extensively used for commercial and governmental applications and substantially extend the range of distributed information systems. The currently deployed satellite networks have mainly been dominated by transponded architectures without any digital processing in satellites. A ground terminal essentially uses the satellite to reflect its transmission to the coverage area (beam) containing the destination and often also the transmitting terminal. This traditional transponded single beam satellite transport, though potentially supporting large coverage areas especially with Geo-synchronous satellites, has severe capacity and connectivity limitations.

\author{
Lisa Orr, Syed Ali \\ Defense Information Systems Agency (DISA) \\ Falls Church, VA
}

Recently, regenerative satellite architectures have incorporated digital processing within the satellite, thus enabling the use of technologies such as onboard resource control and packet processing for innovative networking capabilities. Besides expanding system capacity, these architectures also facilitate true full-mesh (single hop) connectivity across all ground terminals. Standardized by ETSI [1] and TIA [2] for Ka band usage, these RSM-A architectures are now an integral part of pioneering satellite network services for enterprises and consumers. The key features of RSM-A architectures, including packet processing and bandwidth-on-demand function hosted by the satellite, facilitate a responsive platform for providing IP packet services with differentiated QoS. The RSM-A architectures can, for example, send ad hoc intelligence information directly to the warfighter when and where needed, and concurrently gather strategic or special intelligence raw data from theaters. Tactical users on the ground can simultaneously monitor and direct sensors while receiving processed intelligence data. With advanced operations management, long lead mission planning can be accelerated by providing direct feedback to the SATCOM managers.

Packet processing in the satellite is symbiotic with the use of multiple spot beams in both uplink and downlink, leading to an order of magnitude capacity gain (because of the spatial frequency reuse). The use of a number of discrete satellite demodulators and a ground programmable mapping between the demodulators and the beams further enhances dynamic capacity configuration for the system. These advanced satellite capabilities can now easily be used by a wide variety of end user applications with the IP protocol as the common denominator for net-centricity. The RSM-A systems, though appealing, are a novelty with respect to their operational deployment. As described later in the paper, the RSM-A implementations with commercial heritage have many desirable features to support current and future communication needs for diverse communities including armed forces, homeland security, and disaster management.

From a development perspective, it is important to strike the right balance between the satellite payload complexity and the necessary set of onboard networking functionality. RSM-A incorporates layer 1 (physical) and layer 2 (data 
link) packet networking functions. Implemented mostly in (satellite) digital hardware, RSM-A is supported by onboard software functions for payload control, Media Access Control (MAC) processing, and Bandwidth-onDemand (BoD). With the recent advances, it is now becoming possible to implement within the satellite more advanced features, such as full layer 3 packet routing, at higher network layers. These higher layer functions enable extended policy control and routing possibilities across satellites for enhanced net-centricity. In this paper, we describe that key net-centric advantages are well supported even with an RSM-A system.

This paper focuses on a recent commercial RSM-A implementation and its planned over-the-air validation for specific DoD reach back and range extension scenarios under a net-centric framework. RSM-A thus serves as a stepping stone towards more capable packet satellite architectures currently in the design phase.

The paper is organized as follows. The next section provides a summary of the primary RSM-A features. This is followed by a description of net-centric services offered by a satellite system built with RSM-A. The next section presents a framework for analyzing how these satellite system architectures can be evaluated in a net-centric world. This framework is then applied for RSM-A. We then present two specific scenarios which are of significant interest to defense, homeland security, and disaster management communities. We also identify network configurations that can be used for validation with tests and provide some preliminary results related to a research and development project between DISA and Hughes. To conclude, near-term plans are summarized and some exploratory remarks are made for extending RSM-A testing for defense users.

\section{RSM-A ARCHITECTURE}

The RSM-A architecture is centered around digital packet processing and data link control functions within the satellite and associated interactions, via the air interface, with the ground terminals. The ETSI [1] and TIA [2] RSM-A specifications include physical layer (e.g., channel coding, modulation, radio transmission and reception, frame structure, radio link control, and synchronization), satellite medium access control, satellite link control, broadband satellite multimedia services and architectures, and security specification. Per RSM-A, radio transmission from a transmitting terminal is demodulated and decoded in the satellite to extract the fixed size packets along with their destination MAC address. The satellite packet switch uses the MAC address to place a packet on the destination downlink beam and subsequently the packet is received by the destination terminal. A Time Division
Multiple Access (TDMA) scheme coordinates packet transmissions in the shared space media, where a satellitebased BoD function allocates specific carrier frequencies and timeslots for each transmitting terminal. By controlling the number of timeslots and using a specific digital coding scheme, the $\mathrm{BoD}$ function can adapt to changing user traffic and environmental conditions. In addition, RSM-A prescribes differentiated user data transport packet services for providing QoS to upper layers. Both packet processing and resource control functions are under policy-based ground control for further user priority considerations.

As we describe later, the core RSM-A functionality can be extended and enhanced for a specific system instantiation with value-added capabilities. This includes multiple spot beams providing more capacity with spatial reuse, a configurable satellite demodulator with beam mapping for dynamic capacity allocation [3], policy based network management (faster provisioning and granular Service Level Agreement, SLA) [4]), and differentiated end user IP packet services [5]. Figure 1 shows the distribution of IP network, (packet) control protocol, and packet lower layer functions across satellite, Network Operations and Control Center (NOCC), and terminal segments with respect to the core RSM-A functions.

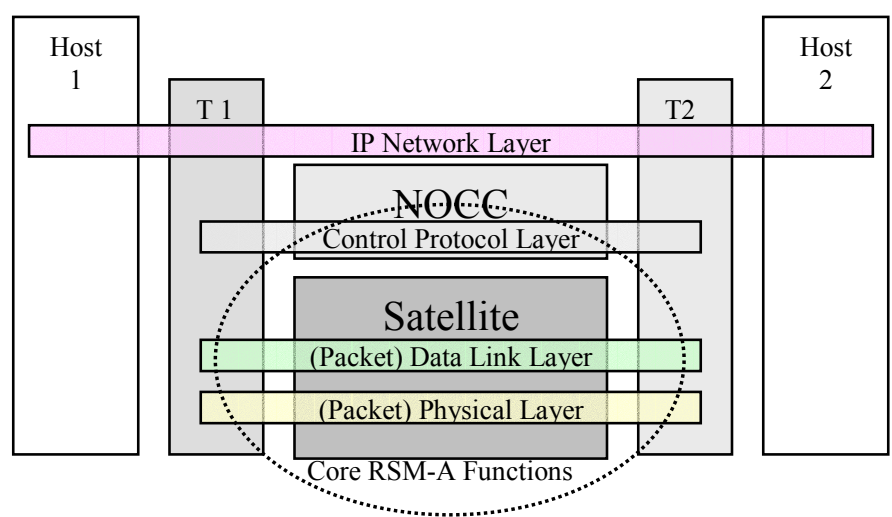

Figure 1 RSM core functions and a system instantiation

The RSM-A architecture supports the always-on concept for IP satellite terminals. A new terminal registers with the satellite network to get the MAC address, security keys, and associated management and terminal user port IP address. A set of basic configuration profiles (for default SLA) are automatically downloaded from NOCC, while specific profiles for additional services and SLAs (such as multicast, DHCP, and connections) are provided selectively based on policy and user mission priorities.

\section{NET-CENTRIC SERVICES}

The first commercial implementation of an RSM-A based satellite system [5] provides IP-based networking for a variety of users. The end user equipment (hosts or routers) 
connect to the satellite terminal with Ethernet cable and interoperate with distant networks using the Internet Engineering Task Force (IETF) standard IP protocols. Besides the basic best-effort service for Internet connectivity, this RSM-A system also provides terminalto-terminal full mesh, multicasting with satellite based packet replication, IP packet Quality of Service (QoS) with differentiation, scalable routing, and VPN services [6]. These net-centric services become possible by leveraging the underlying core RSM-A functions. The RSM-A based systems provide the following (all concurrently with multiple full mesh interactions) on a terminal:

- High performance guaranteed Quality of Service (QoS) needed for voice and video applications.

- On-board Bandwidth-On-Demand (BoD) needed for bursty best-effort (BE) IP traffic.

- Virtual connections (both scheduled and ondemand) with committed resources for critical packet services.

- Multicast with packet replication in the satellite to eliminate unnecessarily replicated uplink transmissions.

- Dynamic scalable routing for each virtual private network (VPN) comprising a set of terminals.

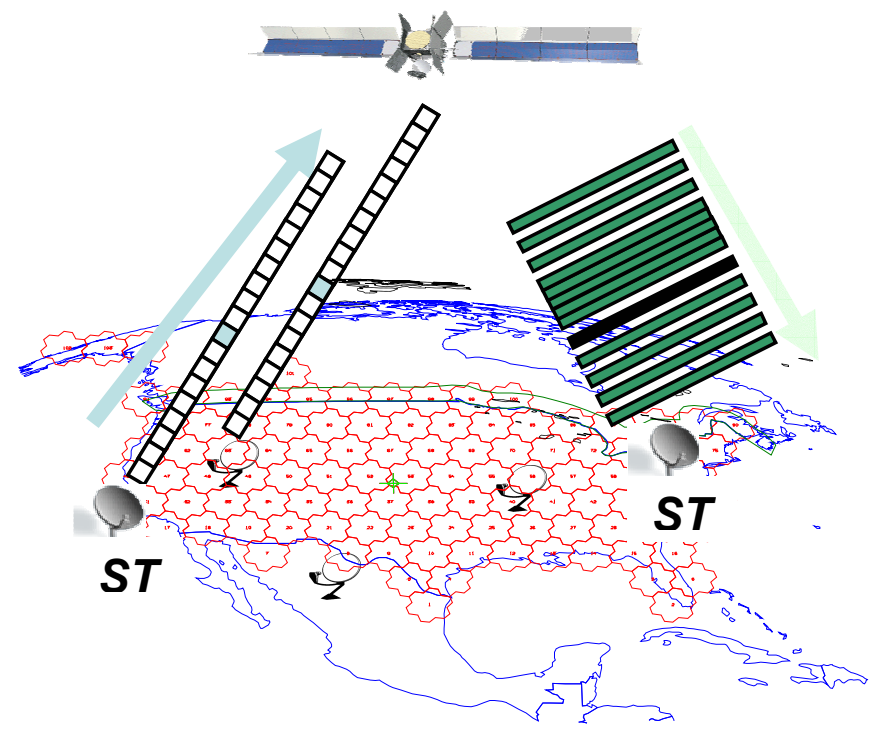

Figure 2 Satellite system based on RSM-A architecture

Figure 2, derived from [6], shows the uplink beam pattern of a next generation satellite networking system that has been commercially deployed in 2008. Further extensions are possible to support the COTM services [7]. Uplink beam locations in this system generally consist of narrow beams (with desirable anti-jamming attributes) in order to provide dramatic increases in system capacity due to frequency reuse among the spot beams. Each uplink beam is itself composed of 7 downlink beams. The on-board digital satellite processor provides separation of the radio uplink from downlink by re-generating the digital signal and thus boosting signal strength [6] at the receiving terminal. Demodulators on the satellite are assigned to particular uplink spot beams to listen to assigned uplink frequency ranges called uplink sub-bands. Uplink antennas and demodulators on the satellite have the ability to separate and differentiate signals from satellite terminals transmitting from the narrow uplink spot beams.

\section{EVALUATION FRAMEWORK}

A framework helps evaluate RSM-A architectures for netcentric services by consolidating various user needs, architectural alternatives, operational costs, and risk factors in a unified fashion.

\begin{tabular}{|l|l|l|}
\hline \multicolumn{1}{|c|}{ Factor } & \multicolumn{1}{|c|}{ Description } & \multicolumn{1}{c|}{ Comments } \\
\hline Capacity & $\begin{array}{l}\text { Traffic at } \\
\text { application and } \\
\text { hierarchical } \\
\text { aggregate levels }\end{array}$ & $\begin{array}{l}\text { Often the primary } \\
\text { factor }\end{array}$ \\
\hline Deployability & $\begin{array}{l}\text { Terminal antenna } \\
\text { size, power, } \\
\text { automated } \\
\text { provisioning, } \\
\text { COTM support }\end{array}$ & $\begin{array}{l}\text { Minimizing } \\
\text { overhead for more } \\
\text { user efficient } \\
\text { satellite system use }\end{array}$ \\
\hline Risk & $\begin{array}{l}\text { Developmental } \\
\text { and operational }\end{array}$ & $\begin{array}{l}\text { Often driven by } \\
\text { functions allocated } \\
\text { to the satellite }\end{array}$ \\
\hline Flexibility & $\begin{array}{l}\text { Adaptive designs } \\
\text { (e.g., dynamic } \\
\text { routing, power } \\
\text { control, capacity) }\end{array}$ & $\begin{array}{l}\text { Responsiveness to } \\
\text { dynamic traffic, } \\
\text { environmental, and } \\
\text { system resources }\end{array}$ \\
\hline $\begin{array}{l}\text { Inter- } \\
\text { operability }\end{array}$ & $\begin{array}{l}\text { With other } \\
\text { satellite and } \\
\text { terrestrial } \\
\text { networks }\end{array}$ & $\begin{array}{l}\text { Standards based } \\
\text { integration of both } \\
\text { end user traffic and } \\
\text { management }\end{array}$ \\
\hline Connectivity & $\begin{array}{l}\text { One-to-one, one- } \\
\text { to-many }\end{array}$ & $\begin{array}{l}\text { Static or dynamic, } \\
\text { scalability }\end{array}$ \\
\hline $\begin{array}{l}\text { Information } \\
\text { Assurance }\end{array}$ & $\begin{array}{l}\text { Privacy, integrity, } \\
\text { authorization }\end{array}$ & $\begin{array}{l}\text { Vs cost of security } \\
\text { implementation }\end{array}$ \\
\hline Manageability & $\begin{array}{l}\text { Policy based } \\
\text { FCAPS } \\
\text { management for } \\
\text { multiple layers }\end{array}$ & $\begin{array}{l}\text { For sustained } \\
\text { optimal } \\
\text { performance and } \\
\text { network SLA }\end{array}$ \\
\hline SM-A EVALA
\end{tabular}

\section{RSM-A EVALUATION}

The SATCOM end users expect superior capacity, connectivity, and coverage from the satellite system in a secure fashion. An easily deployable wide area network allows them to establish communication patterns of multiple kinds including one-to-one and one-to-many involving different kinds of information devices, multimedia applications and information systems. The use of packet switching, multiple beams, and bandwidth-ondemand in RSM-A provides statistical multiplexing for packet network users on top of the spatial reuse of the commercial $\mathrm{Ka}$ band spectrum. RSM-A provides the flexibility to complement this high capacity data and 
control plane functions implemented in layer 1 and layer 2 (see Figure 1) with an IETF-compliant control plane signaling (e.g., RIP, PIM-SM, IGMP, DHCP) implemented in the ground segment (terminals supported by NOCC). An RSM-A system thus provides a packetized data plane without requiring an IETF compliant control plane in the satellite, with resulting risk and cost advantages (especially a few years ago, at the time of RSM-A development). For the end-users, an RSM-A system provides the familiar IP packet services over Ethernet, the most commonly used user network interface.

The framework of the previous section is used to summarize an evaluation of an RSM-A based system for net-centric services in the following table.

\begin{tabular}{|c|c|}
\hline Factor & $\begin{array}{r}\text { RSM-A Evaluation } \\
\end{array}$ \\
\hline Capacity & $\begin{array}{l}\text { Order of magnitude higher (compared } \\
\text { to transponded) capacity with coverage } \\
\text { over continental areas }\end{array}$ \\
\hline Deployability & $\begin{array}{l}\text { Easier with small Ka band antenna, low } \\
\text { power, and automated provisioning and } \\
\text { security key distribution }\end{array}$ \\
\hline Risk & $\begin{array}{l}\text { Smaller with minimal control plane } \\
\text { (software) implementation in satellite }\end{array}$ \\
\hline Flexibility & $\begin{array}{l}\text { Dynamic capacity planning, bandwidth } \\
\text { on demand, and scalable packet routing }\end{array}$ \\
\hline Interoperability & $\begin{array}{l}\text { Net-centric IETF-compliant common IP } \\
\text { protocols across RSM-A system and } \\
\text { other end user networks }\end{array}$ \\
\hline Connectivity & $\begin{array}{l}\text { Both unicast and multicast enabled with } \\
\text { IP control plane joins and leave } \\
\text { signaling at terminals }\end{array}$ \\
\hline $\begin{array}{l}\text { Information } \\
\text { Assurance }\end{array}$ & $\begin{array}{l}\text { Payload control computer assists in } \\
\text { over-the-air distribution of keys to } \\
\text { payload components for capacity } \\
\text { protection and misuse detection }\end{array}$ \\
\hline Manageability & $\begin{array}{l}\text { Management data collection (and } \\
\text { control) from the satellite at layer } 1 \text { and } \\
\text { layer } 2 \text { (complements ground network } \\
\text { management for all layers) }\end{array}$ \\
\hline
\end{tabular}

\section{OPERATIONAL SCENARIOS}

We consider two specific scenarios and show how the RSM-A based systems support these concurrently in an IPbased net-centric fashion. These scenarios subsequently provide a context for tests that will be used to validate the RSM-A architecture. The scenarios are described in a generic fashion to enhance their suitability for a wider application, even though certain terminology from within the DoD domain is used.

\section{Reach Back}

Because of their unpredictability in both time and space, wars and natural disasters defy the fixed coverage of terrestrial networks. Satellite networks provide the reach to deal with these remote networking environments. For these application domains, agents (whether human or sensors) placed in the field (theater of operation, disaster zone, or homeland event zone) have limited or no terrestrially provided connectivity. While executing their mission, these agents have to communicate within some command structure, often going all the way back to headquarters and other sources of information (for example, via Internet or US DoD Global Information Grid). Besides command and control, now these situations increasingly demand extensive information resources. For example, net-centric initiatives enable processing of multisource theater and intelligence data and providing it to the war fighters. These concurrent interactions (unicast, multicast) are bidirectional, high bandwidth, and support significant dynamics with respect to users, network resources, and environment. Interacting with the headquarters over multiple network domains, without compromising QoS and reliability, from the theater of operation and thus fully connecting command and control organizations and information resource centers is the chief hallmark of reach back.

In a restricted way, reach back, as shown in Figure 3, is the only scenario possible with the current generation satellites where a terminal in the field can directly communicate only with a specific Hub (e.g., at the headquarters) and via the Hub to the other terminals. RSM-A provides the required additional capacity, connectivity, and adaptive transport to deal with the dynamics of the environment and next generation net-centric information applications. Netcentric integration over IP simplifies the implementation of the reach back scenario. As shown in Figure 3, end user equipment in theater uses IP to interface with the remote satellite terminal, which is turn uses the RSM-A transport to get connectivity to a high capacity Hub (serving multiple remotes). The Hub (and terminals) has IP router functionality with interfaces to both GIG and Internet.

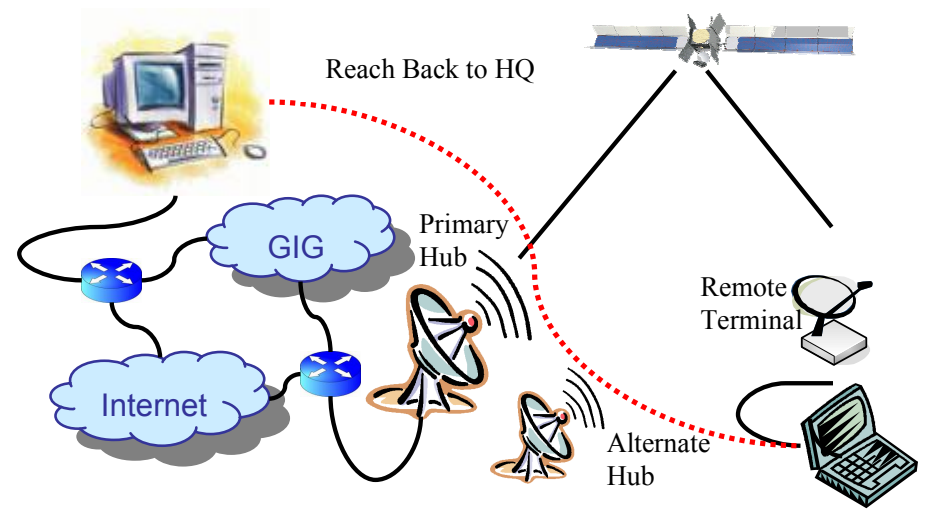

Figure 3 Reach back scenario over multiple networks

As end user net-centric needs require capacity and specific packet QoS for multi-media applications, it becomes necessary that traffic engineering (management plane), 
routing (control plane), and per hop behavior (data plane) for packets are well supported in each networking domain traversed during reach back for a specific mission. A validation of such a reach back scenario has to cover all three aspects of packet networking and over every networking domain included in the path. Since capacity is typically not an issue for the well provisioned terrestrial networks and routing is likely to be more stable as well, it becomes mandatory that the networking capabilities in the three planes are also well supported on satellite networks in an inter-operable fashion. These capabilities are described next as they are also relevant for the more demanding range extension scenario.

\section{Traffic Engineering (Management Plane)}

As mentioned earlier, RSM-A facilitates multi-beam satellite architecture with multitudes of demodulators and flexible uplink beam and demodulator mapping. Packet switching within the satellite allows the downlink to deal with finely granular information chunks (packets) which are destined for a specific downlink cell. This architecture allows a sophisticated capacity planning function [3] to allocate resources for specific geographic regions, organizations, and time durations, using the satellite network. This meshes well with the traditional traffic engineering function for a packet routing network.

\section{Routing (Control Plane)}

The packet routing function for the RSM-A based system is a layer 3 (IP) extension of the basic address resolution function that correlates layer 3 network IP addresses of the terminals with the MAC addresses within the satellite system. This address resolution is enhanced first with policy control for securing access patterns and to implement closed user groups [6]. Thus a specific terminal can inter-communicate with only those terminals in the user group (similar to layer 2 virtual private network) that are compatible with the policy rules specified at the provisioning time for a mission. For the reach back scenarios, the default IP route to a Hub is all that may be needed at every terminal (for simple stub networks). For more advanced communication patterns, a full-mesh (single hop) connectivity requires a richer routing architecture for the terminals. This dynamic routing can import and export terrestrial routes at each terminal with respect to the connected end user terrestrial network. These routes are then exchanged with a Route Server (dedicated for the VPN the terminals belong to) that is provided by a specific terminal (shown in Figure 4). The RS terminal processes all routes and provides optimal routing information to all other terminals in the VPN. By using the multicast feature of the satellite system, this RS architecture avoids control plane flooding needed for some commonly used IGP routing protocols (such as OSPF) for terrestrial networks, while still preserving IP standardscompliance with the end user networks.

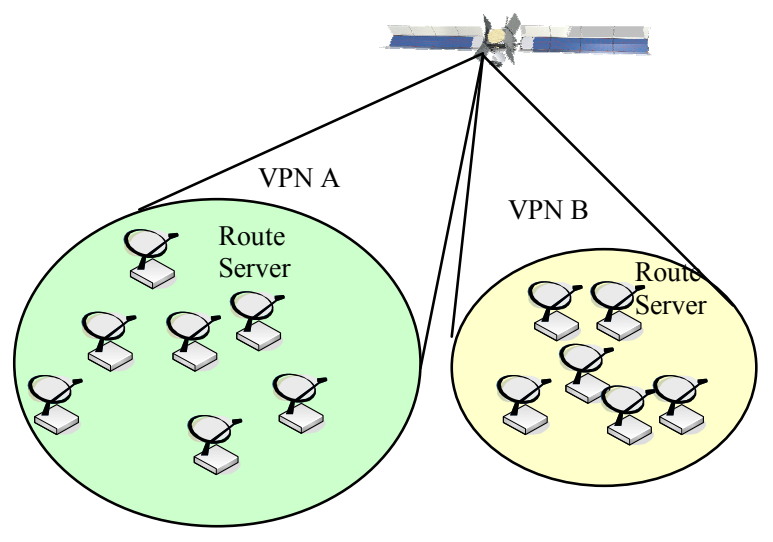

Figure 4 Route server architecture for RSM-A systems

Per Hob Behavior (Data Plane)

The net-centric architectures can use Differentiated Services for QoS. Signaled services, such as RSVP, are less commonly deployed and currently seem problematic from an interoperability perspective. RSM-A can leverage the packetized transport across the air interface to provide differential treatment to packets in terminals and also the satellite packet switch. Packet policing, queuing, and scheduling can now be universally done at all segments of a satellite system. This provides fine control at ingress, uplink, downlink, and egress of the system. With value added visibility into capacity allocations [3], RSM-A systems can go beyond Diffserv to provide specific QoS at the packet flow level. The flow admissions are governed in both scheduled and on demand fashion, ensuring that sufficient resources are available in the system so that every packet in the flow gets adequate resources at radio transmission and packet queuing and scheduling opportunities. On-demand connections (OC) can optionally be triggered by end user application signaling such as RSVP or simply user data packet DSCP marking, source/destination IP addresses, and port numbers. This flow-specific information is stored as actions in the packet classifier rules of the terminals as part of their configuration for responsive policy enforcement.

\section{Range Extension}

Range extension is a demanding scenario for satellite networks. It is intended to extend the reach of a user network within a theater in a seamless fashion. The satellite networks are used in conjunction with other networks including terrestrial wireless for specific missions. For DoD and other tactical operations, it is quite likely that the end users have multiple transport technologies to reach other members of their group. In one scenario, a tactical group could comprise multiple vehicle-mounted and stationary networking nodes each connected to others using mobile ad hoc network 
(MANET) based on the IP protocol. The dynamics associated with mobility and link impairments can be supported with the use of a packet routing IGP protocol. Thus the routing nodes in the tactical group behave as a tightly-knit single network in a single Autonomous System (AS). This communication pattern among IP routers under a single administrative domain is well understood, supported, and widely deployed by diverse enterprises. A tactical deployment with line of sight (LOS) wireless connectivity providing adequate bandwidth can continue its tactical mission well. However, continued LOS connectivity cannot be guaranteed forever especially because of the environmental conditions, geographical terrain (e.g., a hill obstructing LOS), and urban restrictions. Under these compromised conditions, a more ubiquitous coverage from a satellite complements the capabilities of the terrestrial networks.

A satellite network can heal the partitioning that is caused by a compromised LOS connectivity. Because of the very nature of these tactical missions, putting the partitioned network back into a single whole needs to happen automatically and rapidly. The net-centric IP routing architectures support this need. The RSM-A systems, with full-mesh IP connectivity, scalable Route Server based routing and support for IGP such as RIP ensure that packet paths are dynamically maintained based on appropriate metrics (Figure 5).

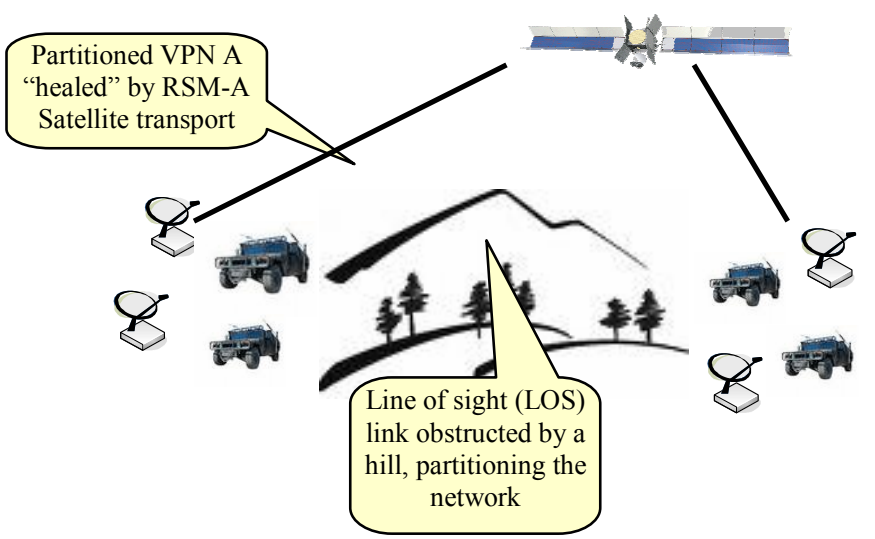

Figure 5 Range extension with RSM-A dynamic routing

When a terrestrial route becomes unavailable because of the LOS breakage, dynamic routing kicks in, routers are marked unavailable. Updated routing information is rapidly propagated both within the terrestrial and satellite segments to ensure that alternate paths become known to all networking nodes for the tactical group. RSM-A can support multiple range extension scenarios concurrently by using a VPN (with its dedicated route server) for each such user group without requiring the user networks to modify their IGP routing architecture.

\section{RSM-A VALIDATION FOR OPERATIONAL SCEANRIOS}

A systematic validation of RSM-A requires designing objective experiments comprising end user needs, packet networking capabilities, network provisioning characterization, and packet QoS and control plane measurements. The work reported here is a precursor to an ongoing research and development initiative between DISA and Hughes for RSM-A which was recently formalized. The overall objective is to form a comparison between the DoD net-centric SATCOM requirements with the RSM-A capabilities. This will determine which DoD requirements RSM-A systems can fulfill. The test data should also be useful in further risk mitigation and system design for the next generation net-centric government satellite systems supporting IP networking.

The end product of this collaborative project should allow the reader to clearly understand which DoD requirements RSM-A systems can satisfy and what analysis method or test metrics were used for such determination. The project will evaluate at a minimum applications performance (e.g., VOIP, FTP, etc.), network operations, and management functions, GIG integration considerations, information assurance, effectiveness of dynamic resource allocation and IP gain. The RSM-A systems may be evaluated on the basis of analysis of design, demo and by test.

Experiments will include the use of satellite terminals which support industry-standard IP and Ethernet for user network interfacing and wide interoperability with current and future DoD networking systems including the GIG. Application performance measurements will involve satellite based packet processing, packet replication, and full mesh capability of system (terminal to terminal connectivity without requiring a central hub).

Terminal service provisioning will be studied with the use of web based service management system. Dynamic resource allocation will include the use of satellite-based bandwidth-on-demand capability and ground-based connection control. Always-on features for the future DoD satellite architectures will be studied with respect to a satellite terminal that, after physical installation, autoregisters and become immediately operational. All configuration, software, and security keys are automatically distributed by the NOCC to the terminal based on the desired services specified via web.

The capability of an RSM-A system relative to the DoD requirements will be determined via witnessed testing and analysis. Performance parameters of interest include but are not limited to FTP, VoIP, Video, IA, NETOPS, 
network management, network defense, dynamic resource allocation and IP gain performance, QoS, and performance under stressed/loaded environments.

This section provides some representative results. A more comprehensive study is in progress (many results expected by the end of 2008). The early results were obtained from a test configuration that was also used for a DoD conference in Florida in May 2008. The following table summarizes the salient features of the RSM-A networking capabilities and their relevance for the two broad scenarios described earlier.

\begin{tabular}{|l|l|l|}
\hline \multicolumn{1}{|c|}{$\begin{array}{c}\text { RSM } \\
\text { Feature }\end{array}$} & \multicolumn{1}{|c|}{ Reach Back } & Range Extension \\
\hline $\begin{array}{l}\text { Traffic } \\
\text { Engineering }\end{array}$ & $\begin{array}{l}\text { Bandwidth for } \\
\text { volume traffic }\end{array}$ & $\begin{array}{l}\text { Bandwidth for rate } \\
\text { traffic }\end{array}$ \\
\hline Routing & IP routing and NAT & $\begin{array}{l}\text { Point to point } \\
\text { routes and Route } \\
\text { Server }\end{array}$ \\
\hline $\begin{array}{l}\text { Per Hop } \\
\text { Behavior }\end{array}$ & $\begin{array}{l}\text { Delay, loss ( for } \\
\text { best effort or BE } \\
\text { traffic) }\end{array}$ & $\begin{array}{l}\text { Delay, jitter, loss } \\
\text { (on-demand } \\
\text { connection or OC) }\end{array}$ \\
\hline
\end{tabular}

The numerical values in the next three tables are subject to revision based on actual testing and detailed analysis. The data rate range for these tests, especially those using ondemand connections, is $0.128-1.5 \mathrm{Mbps}$.

Management Plane
\begin{tabular}{|l|l|l|}
\hline \multicolumn{1}{|c|}{ RSM-A Feature } & \multicolumn{1}{c|}{ Objective } & Validation \\
\hline Capacity Planning & $<4$ hours & Demo \\
\hline $\begin{array}{l}\text { API transaction } \\
\text { between NOCC } \\
\text { and Service } \\
\text { Provider }\end{array}$ & $\begin{array}{l}<1 \text { minute (specific } \\
\text { profile for a terminal) }\end{array}$ & Test \\
\hline $\begin{array}{l}\text { Remote Use of } \\
\text { Management GUI }\end{array}$ & $\begin{array}{l}\text { Access over } \\
\text { terrestrial and satellite } \\
\text { network }\end{array}$ & \\
\hline
\end{tabular}

\begin{tabular}{|l|l|l|}
\multicolumn{1}{|c|}{ Control Plane } \\
\hline \multicolumn{1}{|c|}{ Routing } & \multicolumn{1}{c|}{ Objective } & \multicolumn{1}{c|}{ Validation } \\
\hline New Route & $60-180$ seconds & (Future) Test \\
\hline $\begin{array}{l}\text { Withdrawn } \\
\text { Route }\end{array}$ & $60-180$ seconds & (Future) Test \\
\hline Scalability & 1000 terminals / VPN & Design \\
\hline
\end{tabular}

\begin{tabular}{|l|l|l|}
\hline $\begin{array}{c}\text { Per Hop } \\
\text { Behavior }\end{array}$ & \multicolumn{1}{|c|}{ Objective } & Validation \\
\hline BE Bandwidth & $1.5 \mathrm{Mbps}$ & Test \\
\hline BE Delay (1 hop) & $\sim 500 \mathrm{~ms}$ (normal op) & Test \\
\hline BE Loss & 1 in 1000 & Test \\
\hline OC Bandwidth & $1.5 \mathrm{Mbps}$ & Test \\
\hline OC Delay (1 hop) & $350-480 \mathrm{~ms}$ & Test \\
\hline OC Jitter & $15-40 \mathrm{~ms}$ & Test \\
\hline OC Loss & 1 in 10000 & Test \\
\hline
\end{tabular}

\section{CONCLUSION}

This paper provides a high level description of the next generation RSM-A Ka band technology and a compliant satellite IP network deployed in 2008 and how they can support current and evolving DoD requirements, including reach back and range extension scenarios. With some preliminary experimental results, this paper sets the stage for a more detailed net-centric evaluation of the RSM-A systems which is currently under progress. This evaluation will address both the validation of current implementations of the existing RSM-A based systems, and also provide insights into how RSM-A can be extended to better support the war fighter needs. Besides high capacity net-centric COTM capability which is already described for RSM-A [7], other possibilities include a programmatic integration of RSM-A network management with DoD manager-of-manager architectures.

Another important area for future work would be to investigate and systematically validate the evolving class of more resilient IP based satellite applications for RSMA. These include the latest TCP and HTTP performance enhancement proxies designed for the satellite transport. It will become possible to migrate many more legacy and future applications after validating them for both reach back and range extension scenarios over SATCOM with regenerative mesh capability.

\section{REFERENCES}

1. European Telecommunications Standards Institute (ETSI) Technical Specification 102188 and 102 189, Satellite Earth Stations and Systems; Regenerative Satellite Mesh(RSM-A) air interface, 2004.

2. Telecommunications Industry Association (TIA) published TIA-1040.1.07 - Regenerative Satellite Mesh-A (RSM-A) Air Interface.

3. D. Whitefield and R. Gopal, "Capacity Enhancement with Dynamic Resource Management for Next Generation Satellite Systems," MILCOM 2005.

4. G. Totsline, R. Gopal, "On Managing Intelligent Satellite Networks - An Evolutionary Approach in Policy Based Distributed Management," MILCOM 2005.

5. R. Gopal et. al, "Technology Readiness of Future Generation Networks Leveraging Regenerative Satellite Mesh Architecture - A SPACEWAY Perspective," MILCOM 2006.

6. D. Whitefield, S. Arnold and R. Gopal, "SPACEWAY Now and In the Future: Onboard IP Packet Switching Satellite Communication Network," MILCOM 2006.

7. S. Arnold, D. Whitefield, and R. Gopal, "Mobile Communications in a Geosynchronous Regenerative Satellite Mesh (RSM) System," MILCOM 2006. 